\title{
LA INSERCIÓN PROFESIONAL DE LOS
}

GRADUADOS EN CIENCIAS DE LA

EDUCACIÓN: PANORAMA EN

IBEROAMÉRICA Y CONFIGURACIONES

EN ARGENTINA

\section{Employability of graduates in Sciences Education:}

similarities and differences in Ibero-America

$M^{a}$ Eugenia Vicente*

\section{RESUMEN}

El campo profesional de las Ciencias de la Educación es caracterizado como heterogéneo, difuso y fragmentado debido a las tendencias de diversificación de los espacios de inserción profesional en Iberoamérica. En este sentido, el presente estudio pretende aportar datos sobre las características comunes y diferentes de los mecanismos de inserción profesional y del campo profesional de las Ciencias de la Educación en el plano internacional. Para ello, se lleva a cabo un análisis comparativo de fuentes secundarias a partir de las siguientes dimensiones: I) la situación de empleabilidad, II) el tiempo que tardaron en conseguir el primer empleo, III) las formas de ingreso a los espacios de trabajo IV) la formación recibida.

\footnotetext{
* Universidad Nacional de La Plata (Argentina).
} 
PALABRAS CLAVE: Ciencias de la Educación; Inserción Profesional; Campo Profesional; Graduados.

\begin{abstract}
The professional field of Sciences Education is characterized as heterogeneous, diffuse and fragmented due to diversification of the employment spaces in Iberoamerica. The present study aim is to provide data on the common and different features of mechanisms employability and professional field of Educational Sciences at the international level. For that purpose, this study developed a comparative analysis of secondary sources around the following dimensions: I) the situation of employability, II) the time that graduates get the first job, III) the forms of access to the employment spaces, and IV) training.
\end{abstract}

KEY WORDS: Sciences Education; Employability; Professional Field; Graduates.

$* * * * *$

\title{
INTRODUCCIÓN
}

Desde el enfoque de la sociología de las profesiones, Tenti Fanfani y Campo (1990) y Dubet $(2006,2007)$ señalan un conjunto de transformaciones ocurridas en los campos profesionales durante los últimos tiempos y que permiten abordar el campo particular de las Ciencias de la Educación en su complejidad. En primera instancia, la aparición de nuevos destinatarios o clientes en el contexto ocupacional ha modificado el ejercicio profesional. La docencia, las carreras científicas, las humanidades tienen como clientes potenciales exclusivos a las organizaciones o a las comunidades, o grupos sociales, en todo caso a entidades colectivas. El rol del profesional se hace cada vez más complejo, en la medida en que se inscribe dentro de espacios sociales complejos, lo cual obliga a interactuar con colegas, con otros especialistas, con administradores, y con clientes colectivos cuyos problemas no aparecen delimitados en forma clara e inmediata. Así, han cambiado profundamente las condiciones objetivas del diagnóstico como de la solución de los problemas básicos. Para el caso de las Ciencias de la Educación, existe una mayor complejidad de los vínculos profesionales e institucionales para sostener las propuestas educativas en la actualidad. La presencia de la acción pedagógica en espacios sociales mayores a los del sistema educativo, o el ámbito escolar o académico puntualmente, ha constituido una extensión de las relaciones entre los graduados y profesionales de otros campos del conocimiento como los trabajadores sociales, los arqueólogos, los antropólogos, los geólogos, los astrónomos, los físicos, los matemáticos, los biólogos, los geógrafos, los comunicadores visuales, los actores, los músicos, los psicólogos (VICENTE, 2013). 
En segunda instancia, los roles laborales de los profesionales se hacen cada vez más variados a medida que la sociedad se hace más diferenciada y compleja. Esta complejidad genera una serie de tendencias hacia la diferenciación de las profesiones en especialidades y sub-especialidades. Esto acrecienta la disputa por el problema de las jurisdicciones entre profesiones y sus respectivas especialidades, mantienen relaciones de competencia para la realización de tareas específicas (SARFATTI LARSON, 1989). En este sentido, Fernández (1989) y Furlán (1988) afirman que las Ciencias de la Educación como profesión se encuentra en encrucijada; las luchas y disputas interprofesionales por la dominación y posesión del campo profesional han tenido un carácter permanente. En cierta medida, su ambigüedad conceptual, social, profesional, debilita a la profesión en su lucha y disputa por el dominio y la hegemonía de las prácticas profesionales de la educación. Es a partir de esa multiplicidad de enfoques y posturas que el profesional de las Ciencias de la Educación resulta algo difuso y confuso. Por consiguiente, los empleadores no necesariamente encontrarán en esta ella algo útil o esencial para ser tomado en cuenta. Más aún, el hecho de que desde hace algunos años se hayan creado en las universidades programas para formación de profesionales en Ciencias de la Educación generó un grupo de profesionales aprovisionados de discurso pedagógicos de diversa índole y calidad, que por no tener amarrada una zona fija de captación laboral, se interrogan frecuentemente acerca de su identidad.

En tercera instancia, durante mucho tiempo el trabajo sobre los otros fue concebido, tanto por quienes lo llevaban a cabo como por quienes lo observaban, interpretaban y lo dirigían, como un proceso institucional; procedía de un programa institucional. No obstante, Dubet $(2006,2007)$ reconoce que en la actualidad existe una descomposición de elementos y representaciones que el programa institucional tuvo la capacidad de integrar en un sistema percibido como más o menos coherente. Los valores perdieron su unidad; la vocación choca contra los requerimientos de eficacia profesional, contra los constreñimientos de organizaciones más lábiles y más complejas, y la creencia en una continuidad entre socialización y subjetivación ya no resulta evidente. Cuanto más se alejan los actores de este programa institucional, el trabajo sobre los otros se presenta cada vez menos como el cumplimiento de un rol y cada vez más como una experiencia compuesta.

Para el caso particular del campo de las Ciencias de la Educación, la práctica de formación docente y el espacio del sistema educativo contribuyeron a la institucionalización de esta disciplina. En la actualidad el campo profesional se constituye de múltiples referencias socio-profesionales por fuera del sistema educativo y de la profesión docente, poniendo en cuestionamiento la institucionalización de las Ciencias de la Educación. En los orígenes de este campo, los docentes eran los destinatarios principales de las prácticas de intervención de los graduados, pero en la actualidad no sólo son los docentes y escolares, sino los trabajadores, los ciudadanos en general, los visitantes de 
museos, los artistas, los empresarios, los adultos mayores, los pacientes de hospitales (VILLA, 2011; ARMENGOL, 2005; GUELMAN, 2007).

En este marco, la presente investigación tiene el objetivo de aportar datos que enriquezcan el conocimiento sobre el campo profesional de las Ciencias de la Educación en la actualidad, al mismo tiempo que contribuya a analizar la situación profesional de los graduados en Iberoamérica. Para ello, este estudio realiza un análisis comparativo de las características de inserción profesional de los graduados en diferentes países durante la última década.

\section{MÉTODO}

Para llevar a cabo el objetivo propuesto, se realizó una revisión de los estudios e investigaciones sobre la inserción profesional de los graduados en Ciencias de la Educación de universidades ubicadas en diferentes países de Iberoamérica. La búsqueda de dichos estudios se realizó en las bases de datos de: I) catálogos en línea (BIB y REVIS) de Flacso; II) Memoria Académica: repositorio digital de revistas, boletines, tesis y audiovisuales; III) E-BIBIOTECA: acceso a la producción documental y bibliográfica en Ciencias Sociales sobre la Región Andina y América Latina en general; IV) EBSCO reserch database; V) JStor, principalmente las bases de SocINDEX, Professional Development Collection y Education Resource Information Center (ERIC), Portail de revues scientifiques en sciences humaines et sociales (PERSEE), SCIelo, Dialnet; VI) Actas de Congresos o encuentros sobre educación y trabajo en Ciencias de la Educación.

Los datos analizados de los estudios seleccionados proveen información sobre los graduados en Ciencias de la Educación de las universidades de: Coimbra (Portugal), Universidad de Minho (Portugal), Universidad de Barcelona (España), Universidad Católica Boliviana (Bolivia); Universidad Evangélica de Bolivia (Bolivia); Universidad Gabriel René Moreno (Bolivia); Universidad de Tlaxcala (México), Pontifícia Universidade Católica do Rio Grande do Sul (Brasil), Universidad Nacional de Salta (Argentina), Universidad Nacional de La Plata (Argentina), Universidad Nacional de Cuyo, Universidad Nacional de San Martin (Argentina), Universidad Nacional de Luján (Argentina), Universidad de Buenos Aires (Argentina), Universidad Nacional de Jujuy (Argentina).

Los países han sido nucleados en tres grupos: Europa, América Latina y dentro de ésta las características particulares del campo en Argentina. Este último caso se ha separado del resto de los países de América Latina para lograr equilibrar el análisis, debido a la gran cantidad de datos hallados sobre los graduados en Ciencias de la Educación en universidades argentinas. Finalmente, las variables utilizadas para analizar comparativamente el campo profesional de las Ciencias de la Educación en cada grupo de países son las siguientes: I) la situación de empleabilidad, II) el tiempo que tardaron en 
conseguir el primer empleo, III) las formas de ingreso a los espacios de trabajo IV) la formación recibida.

\section{EL PANORAMA EN EUROPA: ESPAÑA Y PORTUGAL}

En España y Portugal $^{1}$, los graduados en Ciencias de la Educación se encuentran insertos en cinco espacios de actuación profesional diferentes (ALCOFARADO et. al., 2007; SILVA, 2001; VENTURA BLANCO, 2005): un primer espacio referido a las instituciones de solidaridad social como los espacios de tiempos libres, las residencias para la tercera edad, los centros de apoyo, los centros de día y la Cruz Roja. Un segundo espacio de inserción referido a las instituciones de enseñanza pública no superior como el magisterio, la formación docente para escuelas de pre-escolar, las escuelas de enseñanza básica y de enseñanza secundaria. Un tercer espacio de inserción refiere a la función pública, como por ejemplo la administración escolar, las direcciones locales y regionales de educación, la inspección escolar, los institutos de jóvenes, la gestión en la universidad, en los hospitales y en la seguridad social, entre otros. Un cuarto espacio de inserción se trata de las cámaras municipales, y en quinto lugar, en los espacios de las empresas de productos y servicios.

En relación a la situación de empleabilidad, la mayoría de los graduados se encuentran empleados y algunos de ellos están realizando estudios de posgraduación. Con relación a los graduados desempleados, la mayoría se encuentra en la búsqueda del primer empleo, y un grupo menor no se encuentran trabajando porque se dedican a estudiar a nivel de posgraduación. Según Ventura Blanco2005) el $80,1 \%$ de los graduados fueron empleados antes o durante la realización de los estudios de la licenciatura de Pedagogía 2 . La mitad de las personas que trabajaban antes o durante la carrera reconocen que las funciones y tareas que realizaban estaban relacionadas con la educación, lo cual es un posible indicio de vinculación vocacional entre la trayectoria laboral y la carrera académica de una buena parte de los graduados. De las personas que no realizaban funciones y tareas relacionadas con la educación (casi la mitad), un 81,5\% desempeñan su trabajo en el sector terciario y el $18,5 \%$ en el sector secundario.

En síntesis, la inserción profesional refleja elevados niveles de ocupación en oposición a unos bajos índices de paro e inactividad, con una tasa de ocupación que oscila aproximadamente entre el $85 \%$ y el $90 \%$. Respecto de la contratación de los graduados, la mayoría tienen un contrato temporal en su primer empleo significativo luego del egreso, el resto se distribuyen entre contratos como autónomos y contratos indefinidos. Más de la mitad de los graduados empleados se encuentran en situación de prestación de servicios. En

\footnotetext{
${ }^{1}$ Datos en base a las siguientes muestras: 29 graduados de la Universidad de Coimbra (Portugal), 119 graduados de la Universidad de Minho (Portugal), 141 graduados de la Universidad de Barcelona (España).

${ }^{2}$ La carrera de Ciencias de la Educación en España se denomina Pedagogía.
} 
relación a la satisfacción profesional, la mayoría mantienen una alta satisfacción profesional. Más de la mitad de los graduados reconocen tener un nivel elevado o muy elevado de satisfacción profesional, seguido de un grupo menor de graduados que reconocen tener un nivel medio o bajo de satisfacción profesional.

En relación al tiempo que tardaron en conseguir el primer empleo, los graduados de Ciencias de la Educación se dividen en dos grupos. Por un lado, aquellos que consiguieron empleo luego del egreso transcurriendo cierto tiempo entre ambos momentos. Por otro lado, aquellos graduados que ya estaban insertos laboralmente mientras transitaban por la carrera de Ciencias de la Educación y en su mayoría desarrollan funciones y tareas relacionadas con la educación, actividades que mantienen luego del egreso. Para quienes se insertan posteriormente al egreso, la mayoría lo hacen en menos de seis meses y en algunos casos en menos de un mes. En menor medida, tardan en insertarse entre siete y doce meses, y pocas veces más de un año.

Respecto de los mecanismos o formas de acceso a los empleos, las estrategias utilizadas para la obtención del primer empleo son varias y simultáneas: refieren a los contactos informales que comprende a familiares y amigos, la "candidatura espontánea" que se lleva a cabo a través de la presentación de curriculum vitae en diferentes instituciones, las respuestas a las ofertas laborales, la participación en los centros de empleos, y las invitaciones por parte de los empleadores. Asimismo, otras formas de acceso a los espacios de trabajo son a través de concursos públicos, invitaciones durante las prácticas de la carrera o en la misma institución donde se formaron, y a través de internet. De los métodos utilizados en la búsqueda de empleo, los considerados más efectivos por parte de los graduados son: la utilización de contactos personales a través de padres, familiares o amigos, el uso de internet y el envío de currículum. Como se mencionó más arriba, los tiempos de inserción profesional varían según si los graduados consiguieron su primer empleo durante la carrera (o sea, como estudiantes) o luego del egreso. Quienes se insertaron en el mercado laboral antes de la graduación lo hicieron a través del ofrecimiento directo a los empleadores, seguido de los contactos a través de amigos o conocidos y de la participación en concurso público. Para quienes se insertaron en el mercado laboral luego del egreso, los métodos utilizados en la búsqueda de empleo se llevó a cabo principalmente a través del envío de curriculum y la utilización de internet, y en menor medida, a través de los contactos personales a través de padres, familiares o amigos.

Respecto de la formación recibida, durante la carrera de Ciencias de la Educación la mayoría de las prácticas son llevadas a cabo en instituciones del ámbito sociocultural, en menor medida en el ámbito de la empresa y en último término en el ámbito formal (sistema educativo). Así, el ámbito sociocultural es el más demandado por el alumnado, seguido por el de la empresa, siendo el ámbito formal el menos solicitado. En relación a la formación recibida, los graduados reconocen una importante adecuación entre las habilidades y 
conocimientos prácticos y las necesidades que se derivan de su actual empleo. Al mismo tiempo, reconocen que la formación los preparó lo suficiente para la inserción profesional y utilizan los conocimientos y habilidades adquiridas a lo largo de la carrera en tareas ligadas a su trabajo actual. En relación a la contribución de la formación al trabajo, para quienes se insertaron profesionalmente luego del egreso, la mayoría reconoce que la formación en Ciencias de la Educación contribuyó directamente en la situación actual de empleabilidad. Para quienes se encontraban insertos durante la carrera, en general, la carrera contribuyó a la movilidad laboral. Particularmente, los cambios en el trabajo luego del egreso implicaron mejoras en la remuneración, cambios de funciones y obtención de cargos de mayor jerarquía. En menor medida, quienes declaran que tuvieron movilidad laboral luego del egreso de la carrera de Ciencias de la Educación, son graduados que ejercían la docencia durante la formación y luego del egreso aún continúan en dicho ejercicio.

\section{LA INSERCIÓN PROFESIONAL DE LAS CIENCIAS DE LA EDUCACIÓN EN AMÉRICA LATINA: MÉXICO, BOLIVIA, BRASIL Y URUGUAY}

Los graduados en Ciencias de la Educación de México, Bolivia, Brasil y Uruguay ${ }^{3}$ se encuentran insertos en múltiples espacios de inserción profesional (JUSTINIANO DOMÍNGUEZ, 2006; JUÁREZ HERNÁNDEZ, 2009; MOROSINI et. al., 2011; MARTINIS, 2011) donde, a diferencia de España y Portugal, predomina el sistema educativo en sus diferentes aspectos (escuelas, la gestión del sistema educativo, los institutos de formación docente, la universidad) aunque también se presentan otras opciones, apareciendo con más fuerza que en años anteriores a la última década las propuestas no formales. Ha crecido la demanda para coordinar acciones de formación de Recursos Humanos y de responsabilidad social empresarial en empresas privadas, como así también en los proyectos de educación extraescolar y en los ámbitos de planificación educativa en los organismos rectores de la enseñanza.

En relación a la situación de empleabilidad, los graduados que se encuentran activos laboralmente constituyen el grupo mayoritario. Este dato era de esperar al tratarse de un grupo que ha contado con una profesión e inserción laboral previa. De esta manera, entre los graduados de Ciencias de la Educación existen dos grupos que difieren según posean o no un título docente previo al ingreso a la carrera de Ciencias de la Educación y que impacta en el momento de ingreso al campo laboral en general y en las expectativas profesionales como graduados en Ciencias de la Educación. En relación al grupo de graduados que contaban con título e inserción laboral previa al egreso, se encontraban ejerciendo su profesión inicial (docencia) o realizando un trabajo afín. En el caso de Bolivia

\footnotetext{
${ }^{3}$ Datos en base a las siguientes muestras: 75 graduados de la Universidad Católica Boliviana (Bolivia); 49 graduados de la Universidad Evangélica de Bolivia (Bolivia); 119 graduados de la Universidad Gabriel René Moreno (Bolivia); 128 graduados de la Universidad de Tlaxcala (México), y a los graduados de la PUCRS en Brasil.
} 
éste grupo es el mayoritario en la matrícula de la carrera. Quienes continuaron su formación como licenciados en Ciencias de la Educación, tenían previsto una movilidad laboral después del egreso y realizaban esta formación con el propósito de desarrollar competencias que les permitan un ascenso social. Entre las motivaciones para estudiar mientras trabajaban prevalecen el establecimiento de vínculos para un trabajo posterior y las prácticas profesionales. Por su parte, quienes continúan en el mismo trabajo antes del egreso prevalece la función docente seguida, en una proporción menor, por la dirección. Finalmente, en el grupo que logró movilidad laboral, si bien existe un predominio de la función docente, también aparecen otras funciones como las de consultoría u otras funciones cualificadas, y en menor porcentaje aparece la función de dirección.

Respecto del tiempo en que los graduados tardaron en conseguir el primer empleo, o respecto del momento de inserción profesional, se diferencian dos grupos. Por un lado, quienes durante el período de formación se encontraban ejerciendo su profesión inicial (docencia) o realizando un trabajo afín. Por otro lado, quienes no estaban insertos laboralmente antes del egreso, consiguieron trabajo antes de los doce meses y más de la mitad consiguió trabajo entre uno a tres meses.

En relación a las formas de acceso a los empleos, se presentan dos tendencias: una predominante que es a partir de los mecanismos relacionales, a través de contactos personales como amigos, familiares, maestros y conocidos. En segundo término y en menor medida, se hace uso de los mecanismos propios del mercado de trabajo, donde ponerse en contacto con el empleador por iniciativa propia es el medio para el ingreso. Así, las recomendaciones directas y la red de amigos se constituyen en un elemento importante que favorece la inserción y movilidad de los profesionales. Por lo que respecta a las vías de acceso al trabajo de los profesionales que continúan en el mismo trabajo, prevalecen las vías estatales, ya sea por nombramientos directos o licitación pública. Esto significa que el Estado es el principal encargado de la contratación, tanto de los docentes como de los profesionales en Ciencias de la Educación. En el grupo que alcanzó movilidad laboral dentro del espacio laboral en que ya se encontraba inserto antes del egreso, presentan mayor ponderación las vías de ingreso a través de los concursos públicos, seguida de la red de amigos y las recomendaciones directas. En ambos grupos la red de amigos es un elemento importante a la hora de ingresar a los puestos laborales. Por lo tanto, las redes sociales se constituyen en un factor importante para acceder al trabajo.

En relación a la formación, en general, los graduados son formados para una inserción profesional no orientada únicamente para el espacio de la docencia en instituciones educativas e investigación, sino también en el contexto mayor a la escuela en el marco de la sociedad civil, de los espacios socio-comunitarios, del trabajo y el ocio. Puntualmente en México, la mayoría de los graduados durante la última década participaron en proyectos de investigación, prácticas en empresas y otras instituciones. 
Durante los dos últimos años de la carrera en Ciencias de la Educación se hace énfasis en aspectos que son fundamentales para favorecer una transición hacia el mercado laboral.

Por su parte en Uruguay, pensada la formación desde la producción de conocimiento, la creación del Instituto de Educación y la propuesta de un nuevo Plan de Estudios de la Licenciatura en Educación, actualmente en tratamiento, están dando cuenta de un momento de diversificación también en el caso del campo de la investigación. Las investigaciones actualmente en curso incluyen las variadas perspectivas de la filosofía de la educación; historia de la educación en el Uruguay; abordaje de problemas de la enseñanza desde la perspectiva didáctica; la enseñanza y el curriculum; planificación, las reformas y políticas educativas; las relaciones entre educación, pobreza y desigualdad social; juventud, violencia y educación; género y educación; movimientos sociales y educación; prospección educativa; pedagogía social, escuela y mundo del trabajo.

Para el caso de Brasil, los diseños curriculares (o directrices) nacionales para la carrera parten de reconocer que la acción educativa y el proceso pedagógico se construyen en el seno de relaciones sociales, productivas y étnicas raciales que influencias los principios, conceptos y objetivos de la carrera de Pedagogía. En este marco, en la formación se identifican cinco competencias: conocimiento del área de actuación para la enseñanza, acción didáctico-pedagógica, gestión de procesos educativos, investigación y el ejercicio de la ciudadanía. La enseñanza, los saberes pedagógicos-didácticos y la formación en el ejercicio de la ciudadanía aluden a la docencia en el sistema educativo formal, y en la gestión de procesos educativos se hace presente las funciones orientadas a la gestión de la educación en espacios no formales. Particularmente, la gestión de proyectos se orienta a los saberes sobre la programación, coordinación, ejecución, acompañamiento y evaluación de actividades específicas de la educación, como así también proyectos y actividades educativas no formales. Con relación a la investigación y a diferencia del caso de Uruguay, la formación se orienta a la investigación de la enseñanza y el aprendizaje en el marco de los procesos áulicos. De esta manera, la formación en Pedagogía en Brasil reconoce los procesos y acciones pedagógicos socio-comunitarias a través de la gestión de la educación, de proyectos y propuestas de actividades en espacios sociales por fuera de la escuela.

\section{LA INSERCIÓN PROFESIONAL DE LAS CIENCIAS DE LA EDUCACIÓN EN ARGENTINA}

Como en los anteriores países, en Argentina los espacios de inserción profesional del egresado en Ciencias de la Educación son variados (CARRIÓ DE SCACCIA, 2003; ILVENTO, 2004; JARAMILLO, 2012; MÁS ROCHA, et. al.; 2011; TESTA Y SPINOSA, 
2009; VILLA, PEDERSOLI y MARTIN, 2009; WICHNEVSKY, 2011) ${ }^{4}$. A partir del 2000 que, si bien la docencia en nivel superior se mantiene con fuerte presencia, disminuye la dedicación a la docencia en nivel el secundario y la asistencia psicopedagógica aparece devaluada, y cobran fuerza el asesoramiento pedagógico y la capacitación laboral. Entre los nuevos espacios de inserción en la última década también aparecen aquellos relacionados con las tecnologías educativas, la educación a distancia y el e-learning que, después de los ámbitos de gestión, parecen constituir las segundas nuevas formas de demanda laboral.

En relación a la situación de empleabilidad, y tal como se mencionó anteriormente en el caso de los demás países, se pueden reconocer dos grupos de graduados en Ciencias de la Educación. Por un lado, quienes no presentan título docente previo al ingreso de la carrera y luego del egreso buscan su primer trabajo profesional. Por otro lado, quienes tenían título docente antes de ingresar a la carrera y durante ésta ejercieron la docencia. De esta manera, existe en este grupo una inserción profesional previa al egreso en el ámbito relacionado con el título de Ciencias de la Educación. Con respecto a éste último grupo, la mitad de los ingresantes en la última década que trabajan se dedican a la docencia pudiendo constituirse la carrera de Ciencias de la Educación en instancia de perfeccionamiento, especialización y promoción laboral para los ingresantes que son docentes del sistema educativo. Para el caso de quienes son graduados en Ciencias de la Educación y se dedican a la docencia en nivel superior universitario y no universitario (JARAMILLO, 2012), la mayoría perciben el mercado laboral como limitado y restringido, puesto que consideran que éste presenta obstáculos difíciles de sortear tornando la inserción laboral como una meta difícil de alcanzar. Estos graduados también manifiestan haber depositado muchas expectativas sobre su inserción laboral, las que luego se fueron reduciendo significativamente o no coincidieron con la realidad laboral que les tocó atravesar. En contraposición a esta visión hegemónica, algunos graduados (con y sin título docente previo) piensan que hay una demanda bastante amplia del profesional en Ciencias de la Educación, mientras que otros rescatan el valor de la construcción del propio espacio a través de los denominados "proyectos autogestionados", como por ejemplo la forma en que, junto con un grupo de colegas, generaron acciones para la apertura y el dictado de cursos de capacitación laboral destinado a Auxiliares de Maestras Jardineras.

Al mismo tiempo, si bien gran parte de los graduados se han insertado en el sistema educativo, se observan menos graduados ejerciendo la docencia y otros más insertos en la gestión y el gobierno del sistema en equipos técnicos en la burocracia estatal educativa. Entre las nuevas configuraciones laborales surgidas, aquellas relacionadas con las tecnologías educativas, la educación a distancia y el e-learning, parecen constituir (después de los ámbitos de gestión) las segundas nuevas formas de demanda laboral (VILLA,

\footnotetext{
${ }^{4}$ Datos en base a las siguientes muestras: 35 estudiantes y 6 docentes de la UNSa, 30 graduados de la UNLP, 77 graduados de la UNCuyo, 98 graduados de la UNSAM, 51 estudiantes próximos al egreso de la UNLu, ingresantes a Ciencias de la Educación de la UBA, 12 graduados de la UNJu.
} 
PEDERSOLI y MARTÍN (2009). Al respecto, Ilvento (2004) señala que los graduados durante la década del 2000 evidencian un dilema entre el deseo de desempeñarse en "prácticas no convencionales" en ámbitos no formales de la educación, pero las estrategias de búsqueda se abocan al ámbito formal como "más seguro", en lo que ellos llaman "prácticas convencionales" por ser consideradas como de oferta muy restringida.

En relación al tiempo que tardaron en conseguir el primer empleo, el ingreso temprano al campo laboral educativo a través de la posesión de un título docente previo determina los tiempos de inserción. En correspondencia con el panorama de los espacios profesionales donde se encuentran insertos los graduados, para un parte importante de ellos no representa una modificación en su situación laboral puesto que se trata de quienes ya contaban, antes de iniciar la carrera, con título docente y trabajaban en el sistema educativo (MÁS ROCHA, et. al., 2011). Para quienes no se encontraban insertos antes del egreso de la carrera de Ciencias de la Educación, lograron conseguir empleo entre los 6 y 12 meses.

Respecto de las formas de acceso a los espacios profesionales, las acciones de inserción socio-profesional consideradas más relevantes son aquellas que en primer lugar tienen como escenario laboral la escuela. Así es como la formación complementaria y la inscripción en un registro de aspirantes a la docencia, son las acciones más elegidas. Asimismo, los graduados han conseguido sus primeros empleos a partir de "contactos personales", otros a partir de la presentación de proyectos en instituciones específicas o por la modalidad de pasantías. Una modalidad absolutamente novedosa entre nuestros graduados la representa la inclusión de sus currículum vitae en sitios de internet especializados como son Zona Job, Bumeran.com o redes profesionales como Linkedin (VILLA, PEDERSOLI y MARTIN, 2009).

En relación a la formación en Ciencias de la Educación (CARRIÓ DE SCACCIA, 2003), los graduados encuentran a lo largo de la carrera una escasa práctica profesional, escasa vinculación entre la facultad y la sociedad, y bibliografía desactualizada. Con relación a la formación recibida, consideran estar capacitado o muy capacitado para la docencia, poco capacitado o medianamente capacitado para la investigación; poco capacitado o medianamente capacitado para el asesoramiento pedagógico, poco o nada capacitado para trabajar en equipo, para detectar necesidades sociales y para resolver problemas. Además, los graduados reclaman mayor contacto con la realidad en la que luego desempeñarán su actividad laboral y señalan que los programas, en su mayoría, no contemplan las demandas del medio. No se hace referencia a la función del profesional en Ciencias de la Educación como asesor, como formador de formadores (pares, en instituciones como terciarios y universitarios) ni como Director. Por otro lado, según WICHNEVSKY (2011) en 1986 prácticamente el 70\% de los estudiantes que ingresaban a la carrera de Ciencias de la Educación esperaban que la carrera les brindase la posibilidad de incidir en el mejoramiento del sistema educativo. Visualizaban la necesidad de cambios 
en el sistema educativo y estaban dispuestos a operar sobre el mismo. Este era el motivo más fuerte en la elección de la carrera y confiaban en la posibilidad de incidencia de los profesionales de ciencias de la educación en el sistema. Por otra parte, los ingresantes en los años 2004, 2009 y 2010 si bien manifiestan un importante interés por el mejoramiento del sistema educativo, las posibilidades de tener una buena formación o acceder a un trabajo interesante cobran mayor presencia en la expectativa de esta carrera, presentándose expectativas más individuales que sociales.

Según Cappellaci y Wichnevsky (2011) uno de los dilemas que aparecen fuertemente respecto de la formación de grado, es el de la relación entre teoría y práctica. Tanto los graduados como los estudiantes, señalan la distancia entre la formación y los ámbitos concretos de desarrollo de prácticas educativas. La carrera históricamente concebida como general y teórica, se vuelca preponderantemente hacia el sistema educativo y la escuela como su objeto privilegiado. Sin embargo, los graduados plantean el desconocimiento de las prácticas cotidianas en la formación, postulando que la fuerza de la profesión redunda en entender la educación como una actividad práctica y al mismo tiempo de profunda reflexión crítica. Ilvento (2004) adhiere que se menciona a lo largo de toda la carrera la amplitud del campo, la apertura ambigua y no definida de las posibles inserciones, no obstante las prácticas desarrolladas durante toda la formación y la representación misma que tienen los docentes de la carrera sobre la inserción en un solo lugar posible, hacen que la formación se circunscriba a una perspectiva restringida de la profesión, ausente de la inserción en ámbitos no formales de la educación.

\section{LA INSERCIÓN PROFESIONAL DE LOS GRADUADOS EN CIENCIAS DE LA EDUCACIÓN: SIMILITUDES Y DIFERENCIAS EN IBEROAMÉRICA}

El campo profesional de las Ciencias de la Educación en diferentes países de Europa y América Latina presenta tendencias y características comunes a todos ellos que le otorgan cierta internacionalización pero también diferenciaciones que hacen a una regionalización del campo. Las características de la empleabilidad se presentan como tendencias globales ya que en España, Portugal, Bolivia, Brasil, Uruguay y Argentina, los graduados que se encuentran activos laboralmente constituyen el grupo mayoritario. La inserción profesional refleja elevados niveles de ocupación en oposición a unos bajos índices de paro e inactividad. En relación al tiempo que tardan en insertarse al campo profesional, en general, los tiempos son muy cortos, la mayoría lo hacen en menos de 6 meses y en algunos casos en menos de un mes. O bien, los graduados ya están insertos laboralmente en espacios referidos a educación antes de su egreso de la carrera de Ciencias de la Educación y ésta se constituye en instancia de perfeccionamiento, especialización y promoción laboral para los ingresantes que son docentes del sistema educativo. Estas características demuestran la rápida inserción de la mayoría de los graduados al campo ocupacional en los diferentes países. 
Por su parte, existen diferencias en las formas de ingreso al campo ocupacional y sobre la formación de la carrera en Ciencias de la Educación que la hacen diferente dependiendo del país. En relación a los mecanismos de inserción laboral, en España y Portugal los métodos utilizados en la búsqueda de empleo se llevan a cabo principalmente a través de la presentación de curriculum vitae y la utilización de internet, y en menor medida, a través de los contactos personales por padres, familiares o amigos. A diferencia de los países de América Latina (México, Bolivia, Uruguay, Brasil y Argentina) donde las recomendaciones directas y la red de amigos se constituyen en el medio principal que favorece la inserción y movilidad de los profesionales. En menor medida la presentación de proyectos en instituciones específicas o por la modalidad de pasantías y la presentación de curriculum vitae en sitios de internet especializados en búsquedas y ofertas laborales.

La formación también se presenta regionalizada, en España y Portugal a lo largo de la carrera de Ciencias de la Educación la mayoría de las prácticas son llevadas a cabo en instituciones del ámbito sociocultural, en menor medida en el ámbito de la empresa y en último término en el ámbito formal (en el sistema educativo). Los graduados reconocen una importante adecuación entre las habilidades y conocimientos prácticos y las necesidades que se derivan de su actual empleo. Para quienes se insertaron profesionalmente luego del egreso, la mayoría reconoce que la formación en Ciencias de la Educación contribuyó directamente en la situación actual de empleabilidad. Para quienes se encontraban insertos durante la carrera, en general, la carrera contribuyó a la movilidad laboral. La misma situación se presenta en Bolivia, Brasil, Uruguay y México donde la mayoría de los graduados que ingresaron a la carrera en busca de trabajo se encuentran muy satisfechos con el trabajo actual y reconocen utilizar mucho lo aprendido en la carrera para sus actividades laborales, lo contrario sucede con los interesados en encontrar trabajo. A diferencia de Argentina, donde los graduados encuentran a lo largo de la carrera una escasa práctica profesional y escasa vinculación entre la facultad y la sociedad. Con relación a la formación recibida, la mayoría de los graduados considera estar capacitado o muy capacitado para la docencia, pero poco capacitado para la investigación y el asesoramiento pedagógico. Tanto los graduados como los estudiantes, señalan la distancia entre la formación y los ámbitos concretos de desarrollo de prácticas educativas ya que la carrera históricamente se vuelca preponderantemente hacia el sistema educativo y la escuela como su objeto privilegiado. De esta manera, los graduados se encuentran insertos en espacios laborales por fuera del sistema educativo pero la formación en Ciencias de la Educación no acompañaría a estas prácticas profesionales.

El conocimiento de las características comunes y diferentes entre los países respecto de la formación y el trabajo en Ciencias de la Educación posibilita reconocer ciertas cuestiones. En primer lugar, que en todos los países, entre los graduados existe un grupo de docentes con título previo que trabajan durante la carrera, esto es, que ya están insertos en los espacios de trabajo educativos. En segundo lugar, que en todos los países los graduados 
están insertos en espacios formales como no formales, en diferentes proporciones según sea el país. En tercer lugar, que aquellos países donde la formación de la carrera prepara para los espacios de intervención en el sistema educativo como en los espacios formales, entre los mecanismos de acceso a los espacios de trabajo predominan los institucionales (concursos, presentación de curriculum vitae, presentación de proyectos). En cuarto lugar, que en los países donde la formación está orientada para preparar en las prácticas de enseñanza, investigación y gestión en ámbitos escolares o del sistema educativo, los graduados tienen inserción en dichos espacios principalmente. En quinto lugar, que de los países de América Latina reseñados, Argentina el país con mayor conservadurismo para sumar a la carrera la formación para la inserción profesional en los espacios por fuera del sistema educativo.

\section{REFERENCIAS BIBLIOGRÁFICAS}

ALCOFARADO, L.; SOUSA, C.; MOIO, I.; SIMÕES, J.; RITA, R. y CARVAHLO, V. (2007): Transição para o trabalho de licenciados em Ciências da Educação pela FPCEUC, com estágio curricular na área de Educação de Adultos. Revista portuguesa de pedagogía, 41/3, pp. 375 - 388 .

ARMENGOL, C. (2005): Perfil y competencias de los pedagogos hoy. (Documento curso Pedagogía, España).

CAPPELLACCI, I. y WISCHNEVSKY, J. (2011): Las Ciencias de la Educación en la UBA. Archivos de Ciencias de la Educación, 5, pp. 49-56.

CARRIÓ DE SCACCIA, M. C. (2003): Correspondencia entre el perfil del egresado de la carrera de Ciencias de la Educación y la demanda laboral. Comunicación proyecto Instituto Ciencias de la Educación, Facultad de Filosofía y Letras, Universidad Nacional de Cuyo.

DUBET, F. (2006): El declive de la institución. Profesiones, sujetos e individuos ante la reforma del Estado. (España, Gedisa).

DUBET, F. (2007): El declive y las mutaciones de la institución. Revista de Antropología Social, 16, pp. 39-66.

FERNÁNDEZ, A. (1989): La práctica profesional de la Pedagogía. ANUIES, Revista de la Educación Superior, 18/72.

FURLÁN, A. (1989): La formación del pedagogo. Las razones de la institución en Demarchi, M. y Dumar, D. (Comps.) El campo pedagógico. Cuatro visiones latinoamericanas. Montevideo: Edición de la Revista de la Educación del Pueblo. Serie Documentos Pedagógicos.

GUELMAN, A. (2007): Rupturas en el campo profesional de las Ciencias de la Educación. Ampliación del objeto de la Pedagogía En Vogliotti, A.; de la Barrera, 
S. y Benegas, A. (Comps.) Aportes a la Pedagogía y a su enseñanza. Escriben y debaten los pedagogos. Córdoba: Universidad Nacional de Río Cuarto.

ILVENTO, M. C. (2004): Las representaciones sobre el campo profesional de las Ciencias de la Educación. La incidencia de las prácticas pre-profesionales. Tesis de doctorado. España: Universidad Rovira I Virgili.

JARAMILLO, A. D. (2012): La inserción laboral de los egresados en Ciencias de la Educación de la Universidad Nacional de Jujuy. San Salvador de Jujuy: EdiUnju.

JUÁREZ HERNÁNDEZ, A. (2009): Inserción laboral de los graduados de la Licenciatura en Ciencias de la Educación de la Universidad Autónoma de Tlaxcala. X Congreso Nacional de Investigación Educativa.

JUSTINIANO DOMÍNGUEZ, M. D. (2006): La inserción laboral de los profesionales graduados en ciencias de la educación. Tesis doctoral. Universidad de Barcelona, España.

MARTINIS, P. (2011): Las Ciencias de la Educación en Uruguay. Archivos de Ciencias de la Educación, 5, pp. 37 - 48.

MÁS ROCHA, S. M.; FERRÉ, N. y AGÜERO, K. (2011): Trayectorias profesionales y laborales de los graduados en educación. III Encuentro de docentes, estudiantes y graduados en Ciencias de la Educación, UNSa.

MOROSINI, M. C.; CABRERA, A. y FELICETTI, V. L. (2011): Competências do pedagogo: uma perspectiva docente. Educação, 34/2, pp. 230-240

SARFATTI LARSON, M. (1989): Acerca de los expertos y los profesionales o la imposibilidad de haberlo dicho todo. Revista de Educación, No. Extraordinario: Los usos de la comparación en Ciencias Sociales y en Educación, pp. 198 - 237.

SILVA, B. y MARQUES, F. (2001): Trajectórias de inserção profissional dos licenciados em Educação pelo Instituto de Educação e Psicologia da Universidade do Minho En Gonçalves A. et al. Da universidade para o mundo do trabalho, desafios para um diálogo. Braga: Universidade do Minho.

TENTI FANFANI, E. y GÓMEZ, M. (1990): Universidad y Profesiones. (Buenos Aires, Miño y Dávila).

TESTA, J. y SPINOSA, M. (2009): Las expectativas profesionales de los alumnos próximos al egreso de la Licenciatura en Ciencias de la Educación. (Luján: Universidad Nacional de Luján).

VENTURA BLANCO, J. (2005): El prácticum en los estudios pedagógicos y la inserción laboral. Nuevos enfoques ante el reto europeo. Tesis de Doctorado. (Barcelona: Universidad de Barcelona).

VICENTE, M. E. (2013): Trayectorias profesionales en Ciencias de la Educación: estrategias y prácticas de los egresados en la reconstrucción del campo profesional en Argentina (1970-2012). Tesis de Calificación. Doctorado en Ciencias Sociales, FLACSO Argentina. 
VILLA, A. I.; PEDERSOLI, C. y MARTIN, M. (2009): Profesionalización y campo ocupacional de las Ciencias de la Educación. Archivos de Ciencias de la Educación. $3 / 3,113-128$.

VILLA, A. I. (2011): Pasado y presente de las Ciencias de la Educación. Campo de producción académica y práctica profesional. Revista Archivos de Ciencias de la Educación. 5/5, pp. 13 - 16.

WICHNEVSKY, J. (2011): Los estudiantes de Ciencias de la Educación de la UBA: las cohortes 1986 - 2010. La Plata: VIII Encuentro de Cátedras de Pedagogía de Universidades Argentinas.

\section{PROFESIOGRAFÍA}

\section{$M^{a}$ Eugenia Vicente}

Docente de la cátedra Administración de la Educación de la Facultad de Humanidades y Ciencias de la Educación en la Universidad Nacional de La Plata (UNLP), becaria doctoral del Consejo Nacional de Investigaciones Científicas y Técnicas (CONICET) con lugar de trabajo en el Instituto de Investigaciones en Humanidades y Ciencias Sociales (IdIHCS). Doctoranda en Ciencias Sociales de la Facultad Latinoamericana de Ciencias Sociales sede Argentina (FLACSO). Datos de contacto: Instituto de Investigaciones en Humanidades y Ciencias Sociales, Consejo Nacional de Investigaciones Científicas y Técnicas, Universidad Nacional de La Plata (Argentina). Email: eugevicente@yahoo.com.ar; mevicente@ fahce.unlp.edu.ar.

Fecha de recepción: 20 de abril de 2013.

Fecha de revisión: 7 de mayo 2013 y 3 de diciembre de 2013.

Fecha de aceptación: 5 de mayo de 2014 . 